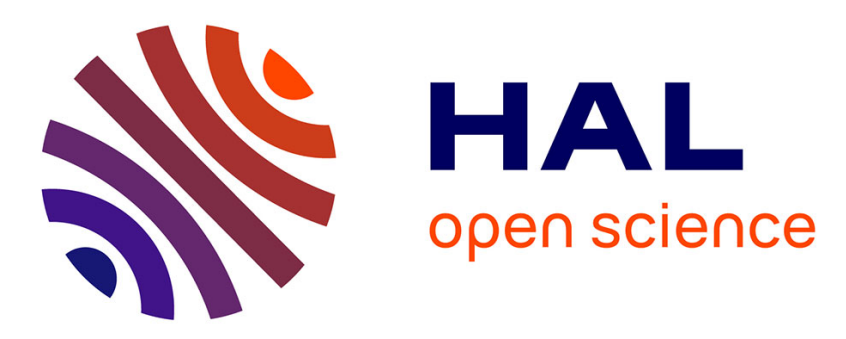

\title{
Integrated optics dissipative soliton mode-locked laser on glass
}

Bertrand Charlet, Lionel Bastard, Jean Emmanuel Broquin

\section{To cite this version:}

Bertrand Charlet, Lionel Bastard, Jean Emmanuel Broquin. Integrated optics dissipative soliton mode-locked laser on glass. SPIE Photonics West, 2011, San Franciso, CA, United States. pp.794108, 10.1117/12.873782 . hal-01965061

\section{HAL Id: hal-01965061 \\ https: / hal.univ-grenoble-alpes.fr/hal-01965061}

Submitted on 7 Oct 2019

HAL is a multi-disciplinary open access archive for the deposit and dissemination of scientific research documents, whether they are published or not. The documents may come from teaching and research institutions in France or abroad, or from public or private research centers.
L'archive ouverte pluridisciplinaire HAL, est destinée au dépôt et à la diffusion de documents scientifiques de niveau recherche, publiés ou non, émanant des établissements d'enseignement et de recherche français ou étrangers, des laboratoires publics ou privés. 


\title{
Integrated optics dissipative soliton mode-locked laser on glass.
}

\author{
Bertrand CHARLET*, Lionel BASTARD and Jean-Emmanuel Broquin \\ Institut de Microélectronique, Electromagnétisme et Photonique-Laboratoire \\ d'Hyperfréquence et Caractérisation, INP Grenoble - Minatec, 3 parvis Louis Néel, 38016 \\ Grenoble Cedex 1, France
}

\begin{abstract}
Mode-lock lasers have been studied a lot in the past years for producing pulses as short as possible. These devices have mostly been realized in bulk optics and they are consequently cumbersome and sensitive to vibrations. There are only a few studies on integrated optics mode-lock lasers, though this technology is very promising because of its stability, compactness and the possibility to integrate several functions on a single chip. In this paper, we present an ion-exchange passively mode-locked laser in dissipative soliton operation. One of the key characteristics of this structure is its mechanical stability. Indeed, no bulk optics is needed because the saturable absorber is hybridized on the top of the waveguide in order to interact with the evanescent part of the guided mode. Indeed, the device that has been obtained is composed of an ion-exchanged single mode waveguide realized in a Neodymium doped phosphate glass. The laser feedback is produced by a Fabry-Perot cavity realized with two multilayers dielectric mirrors stuck on the waveguides facets. We implemented a bis(4dimethylaminodithiobenzil)nickel (BDN) dye included in a cellulose acetate thick film, which presents a saturable absorber behaviour around $1.06 \mu \mathrm{m}$. With this structure, pulses with repetition rates of $3.3 \mathrm{GHz}$ and a single mode output have been measured. Moreover, the use of an autocorrelation set-up allowed us measuring picosecond pulse durations.
\end{abstract}

Keywords: Integrated optics, ion-exchange, mode-locked laser, dissipative soliton

\section{INTRODUCTION}

Ion-exchange on glass, which provides low propagation losses waveguides with a coupling with optical fiber ${ }^{[1]}$ has been a major technology in the field of integrated optics for more than thirty years. It allowed producing numerous passive devices with applications ranging from optical communication duplexers ${ }^{[2],[3]}$ to astronomy ${ }^{[4],[5]}$. Moreover, the use of rare-earth doped glass has allowed realizing active devices like amplifiers ${ }^{[6],[7]}$, continuous wave lasers ${ }^{[8],[9]}$ and Q-switched lasers ${ }^{[10]}$. Nonetheless, there are only few studies on glass integrated optics mode-locked lasers, though this technology is very promising because of its stability, compactness and the possibility to integrate several functions on a single chip. In the last decades, mode-locked lasers for ultra short pulse generation have been studied over a wide range of configurations. This the first analytical theory of passive mode-locking was developed in 1975 by Haus and al. ${ }^{[11]}$. The first mode-locked laser on glass integrated optics, which was working in the soliton operation has been realized by Pudo et $\mathrm{Al} .{ }^{[12]}$. But more recently, dissipative soliton operation has been investigated on optics fiber ${ }^{[13],[14]}$. Unlike classical soliton lasers which operate with opposite signs for Self-Phase Modulation (SPM) and Group Velocity Dispersion (GVD), dissipative soliton lasers operate in normal dispersion regime. The pulse shaping is then determined by the interplay of SPM, GVD, which both shrink the pulse and the filtering effect linked to the finite gain bandwidth of the amplifying medium, which limits the pulse shrinking. Dissipative soliton lasers generate highly chirped pulses, which may be compressed outside the laser cavity. An interesting characteristic of these lasers is their capacity to produce a coherent multi-wavelength output pulse ${ }^{[15]}$. Such a feature is expected to have versatile applications including photonics device characterization and terahertz generation. This kind of modelocked laser operation has only been realized with optical fiber. In this paper, we present a fully integrated

* Send correspondence to Bertrand CHARLET: E-mail : charletb@minactec.inpg.fr, Tel: (+334) 56529488

Integrated Optics: Devices, Materials, and Technologies XV, edited by Jean Emmanuel Broquin, Gualtiero Nunzi Conti, Proc. of SPIE Vol. 7941, 794108 - (C) 2011 SPIE

CCC code: 0277-786X/11/\$18 - doi: 10.1117/12.873782

Proc. of SPIE Vol. 7941 794108-1 
passively mode locked laser made by ion exchange which supports dissipative soliton operation at a wavelength of $1053 \mathrm{~nm}$. The second part of this article is devoted to presenting the device, the realization process with the waveguide design as well of passive characteristics of the device, whereas section 3 deals with the laser finalization and pulses characteristics. Then, section 4 presents the theory on dissipative soliton operation for mode-locked laser.

\section{DECIVE STRUCTURE AND PRINCIPLE OF OPERATION}

The structure of the hybrid mode-locked laser is presented on figure 1, it is composed of three main parts.

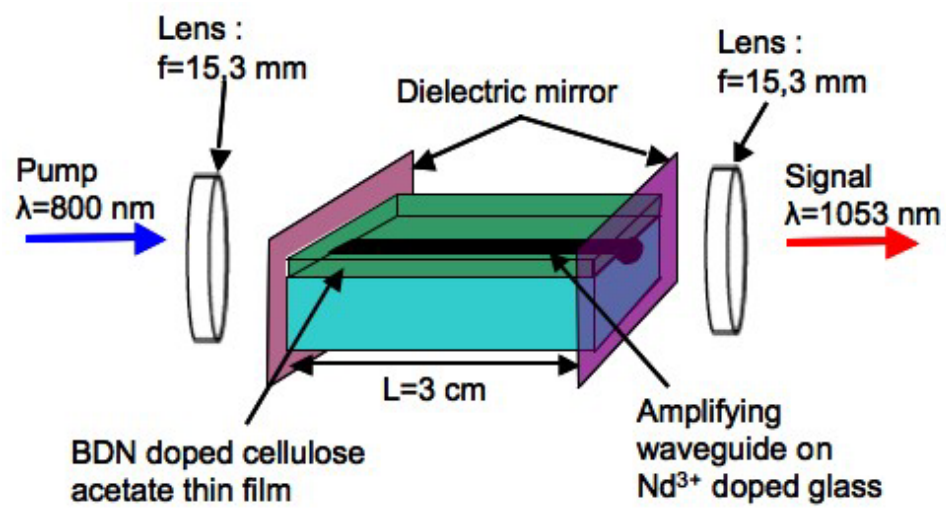

Figure 1: Schematic view of the hybrid mode-locked laser structure

First, it consists of a single mode amplifying waveguide realized by $\mathrm{Ag}^{+} / \mathrm{Na}^{+}$ion-exchange on a $1.5 \times 10^{26} \mathrm{~m}^{-3}$ neodymium doped phosphate glass (IOG1 from Shott). The amplifying waveguide is pump with a $180 \mathrm{~mW}$ Sapphire Titan laser at $\lambda_{\mathrm{p}}=800 \mathrm{~nm}$, which is focused into the waveguide. And acts as an amplifier at $\lambda_{\mathrm{s}}=1053$ $\mathrm{nm}$, which is due to the $\mathrm{Nd}^{3+}$ transitions in phosphate glass. Then, a bis(4-dimethylaminodithiobenzil)nickel (BDN) dye doped Cellulose Acetate (CA) thin film is deposited on the waveguide surface. It acts as a saturable absorber.The interaction between the laser mode and the saturable absorber is made through the evanescence part of the guided mode at signal wavelength as presented on figure 2. Indeed, the CA refractive index is 1,48, which is lower than the glass one of 1,51. This interaction can thus be characterized by the overlap integral between the guided mode and the superstrate. Finally, two dielectric mirrors are stuck on each facet to close the laser cavity. One present a $99.99 \%$ reflectivity at the signal wavelength for the input facet and one of $80 \%$ reflectivity at the signal wavelength for the output one. They both have reflectivity lower than $4 \%$ at pump wavelength

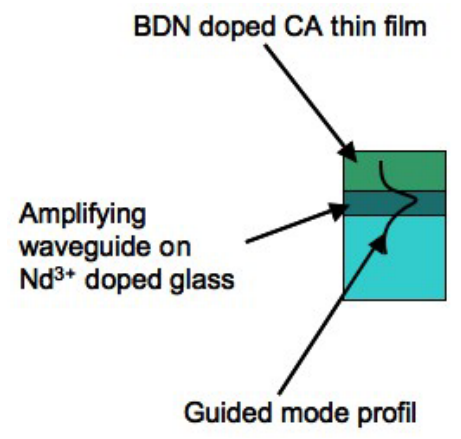

Figure 2: Transverse view of the structure 


\section{WAVEGUIDE}

\subsection{AMPLIFYING WAVEGUIDE REALIZATION PROCESS FLOW}

The amplifying waveguide has been realized on a phosphate glass (IOG-1 from Schott) doped with $1.5 \times 10^{26} \mathrm{~m}^{-3}$ $\mathrm{Nd}^{3+}$ ions with the process flow depicted on figure 3. First, the glass wafer has been degassed during 2 days in a vacuum furnace at $150^{\circ} \mathrm{C}$ in order to remove all the water absorbed on the glass surface. Secondly, a $30 \mathrm{~nm}$ thick silicon layer has been deposited on the wafer surface by RF sputtering. This layer will act as a mask during the ion-exchange step. Then, after lithography, a $3 \mathrm{~cm}$ long aperture has been etched in the silicon layer using a reactive ion etching with a $\mathrm{SF}_{6}$ gas. The next step consists in the ion-exchange that we will design and simulate $\mathrm{n}$ during the next part. Finally, the silicon mask is removed with the same reactive ion etching process than in step 4 and the waveguide facets are cut and polished in order to have low coupling losses.
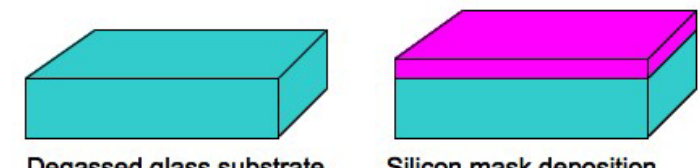

Silicon mask deposition
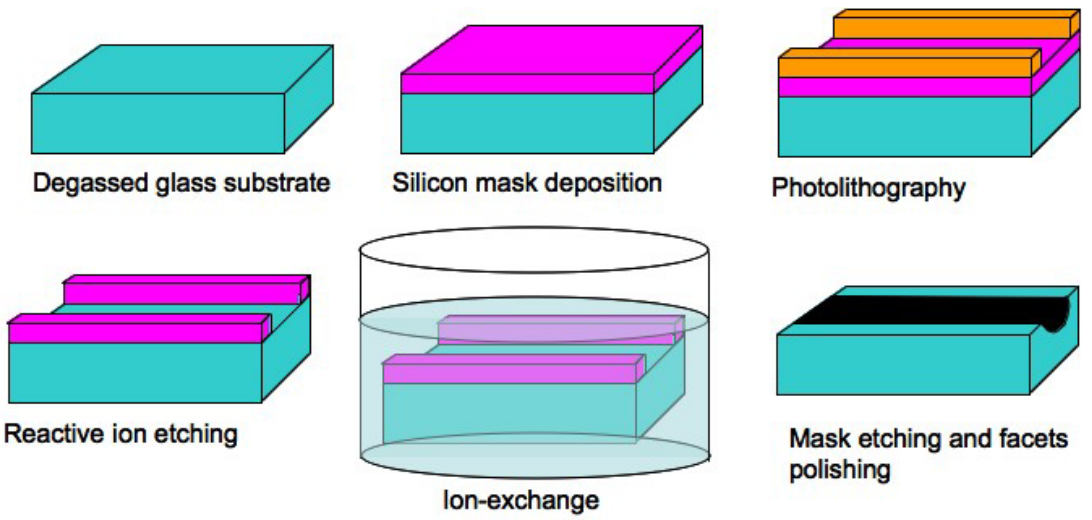

Photolithography

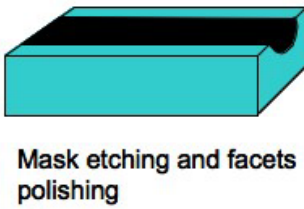

Figure 3: Process flow for amplifying waveguide realization

\subsection{DESIGN OF THE AMPLIFYING WAVEGUIDE}

In order to have a good control on the guided wave confinement and the on the interaction between the evanescent wave and the saturable absorber, the ion-exchange parameters should be design in order to obtain a single mode waveguide at pump and signal wavelength. Therefore, a model of the ion-exchange on phosphate glass is a necessary step before realizing the device.

\subsubsection{ION-EXCHANGE MODELING FOR WAVEGUIDE REALIZATION}

The refractive index variation, which allows the light confinement, is obtained by locally replacing some $\mathrm{Na}^{+}$ ions of the glass substrate by $\mathrm{Ag}^{+}, \mathrm{K}^{+}$or $\mathrm{Tl}^{+}$ions. This doping is made thanks to a diffusion process that can be purely thermal or electrically assisted ${ }^{[1]}$. Combining Fick-Fourrier's diffusion laws with the electro-neutrality one, allows deriving a relation linking the doping ion relative concentration, $c(x, y)$, to the ion-exchange parameters $^{[1]}$ :

$$
\frac{\partial c}{\partial t}=\frac{D_{d o p}}{1-\alpha c}\left[\nabla^{2} c+\frac{\alpha(\nabla c)^{2}}{1-\alpha c}-\frac{e}{k_{B} T H} \vec{\nabla} c \cdot \vec{E}_{\text {ext }}\right]
$$

Where $D_{d o p}$ is the diffusion coefficient of the doping ions, $\alpha=1-D_{d o p} / D_{N a}$ is the Stewart coefficient taking into account $D_{N a}$ the diffusion coefficient of sodium, $\mathrm{H}$ is the Haven coefficient and $\vec{E}_{\text {ext }}$ is the applied electric field. Although $D_{d o p}$ and $D_{N a}$ are considered to be constant in simple models, in our case the mixed alkali effect can be taken into account following the approach proposed by Lupescu et a ${ }^{[16]}$.

For given exchange time, diffusion aperture width, applied electric field and surface concentration, the problem (1) is solved numerically using a finite difference algorithm with a Lax-Wendroff scheme ${ }^{[17]}$. Once the doping ion concentration distribution has been computed, the resulting refractive index distribution is obtained through the simple proportionality relation derived from the model of Huggins $\mathrm{et} \mathrm{l}^{[18]}$. 


$$
n(x, y)=n_{s u b}+\Delta n_{\max } c(x, y)
$$

where $n_{\text {sub }}$ is the refractive index of the glass before the ion-exchange and $\Delta n_{\max }$ is the maximum refractive index variation obtained when $c(x, y)=1$.

This refractive index distribution can then be used as an input for a Scalar mode solver (3D Mode Solver by Optiwave Inc.) to determine the number of guided modes supported by the waveguide and their respective characteristics.

In our case, $D_{d o p}$, which is the diffusion coefficient of silver ions, $D_{N a}$ as well as the mixed alkali parameters have been determined using the m-lines method for different ion-exchanges concentrations.

\subsubsection{ION-EXCHANGE PARAMETERS DETERMINATION}

The amplifying waveguide is made of two ion-exchanges. Both are purely thermals and don't use any electric field. The first one is a Na ${ }^{+} / \mathrm{Ag}^{+}$exchange, which is useful for setting the modicity of the waveguide and the second one is considered as a thermal burying in order to decrease the surface interaction and consequently the propagation losses that are due to surface roughness. The parameters for the first ion-exchange have been set in order to obtain a single mode waveguide at $\lambda_{\mathrm{p}}$ and $\lambda_{\mathrm{s}}$ : a duration of $4 \mathrm{~min}$ in a $20 \%$ silver nitrate $/ 80 \%$ sodium nitrate molten salt bath at $320^{\circ} \mathrm{C}$ and a diffusion aperture of $2 \mu \mathrm{m}$. The glass substrate is the $\mathrm{Nd}^{3+}$ doped IOG1 phosphate glass from Shott. The second step consist in a 1 minute in a $100 \%$ sodium nitrate molten salt bath at $290^{\circ} \mathrm{C}$ ion-exchange, which is keep quick enough to not modify the wave confinement but finely tune the waveguide burying depth for low surface interaction. Indeed, a 5\% interaction between the guided wave and the superstrate has been computed after simulation, which is still present and weak as desire.

The simulated mode field maps has been fitted by gaussians along the horizontal and vertical axes, leading to $1 / \mathrm{e}$ diameters of $6.2 \pm 0.1 \mu \mathrm{m}$ and $1 / \mathrm{e}$ radius of $1.6 \pm 0.1 \mu \mathrm{m}$ for the upper part and $3 \pm 0.1 \mu \mathrm{m}$ for the bottom part

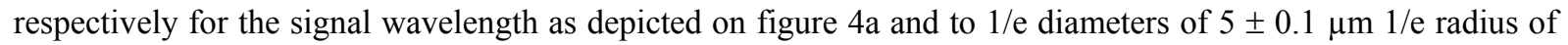
$1.3 \pm 0.1 \mu \mathrm{m}$ for the upper part and $2.1 \pm 0.1 \mu \mathrm{m}$ for the bottom part respectively for the pump wavelength as depicted on figure $4 b$.

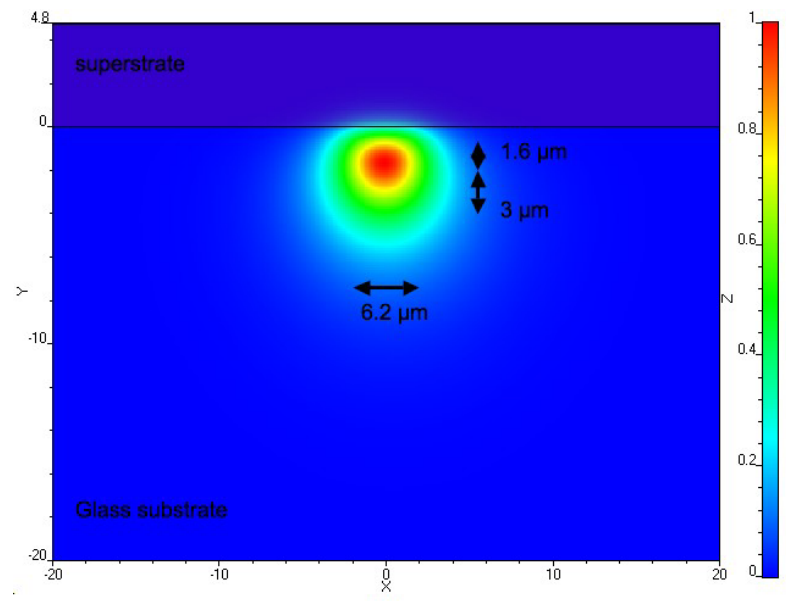

(a)

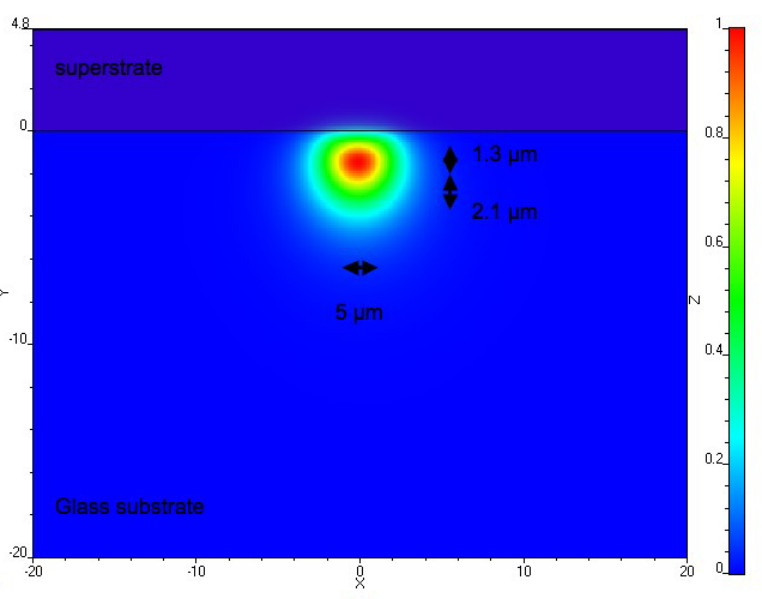

(b)

Figure 4: Field maps of the fundamental mode at $\lambda_{\mathrm{s}}=1053 \mathrm{~nm}$ (a) and at $\lambda_{\mathrm{p}}=800 \mathrm{~nm}$ (b).

\subsection{PASSIVE CHARACTERISTICS OF THE DEVICE}

The mode profiles of the waveguide has been measured at $\lambda_{\mathrm{s}}=1054 \mathrm{~nm}$ and at $\lambda_{\mathrm{p}}=800 \mathrm{~nm}$ by injecting light with a single mode optical fiber and imaging the waveguide output field through a microscope objective on a Silicon CCD camera as it is presented on figure 5. The waveguides are single mode at both wavelengths. The mode intensity map has been fitted by gaussians along the horizontal and vertical axes, leading to $1 / \mathrm{e}^{2}$ diameters of $7.6 \pm 0.7 \mu \mathrm{m}$ and to a $1 / \mathrm{e}^{2}$ radius of $2.2 \pm 0.4 \mu \mathrm{m}$ for the upper part and $2.8 \pm 0.4 \mu \mathrm{m}$ for the bottom part 
respectively at $\lambda_{\mathrm{s}}$ and to a to $1 / \mathrm{e}^{2}$ diameters of $5.8 \pm 0.7 \mu \mathrm{m}$ and to a $1 / \mathrm{e}^{2}$ radius of $2.1 \pm 0.4 \mu \mathrm{m}$ for the upper part and $2.7 \pm 0.4 \mu \mathrm{m}$ for the bottom part respectively at $\lambda_{\mathrm{p}}$. We can notice than the mode fields diameters are close to the theoretical computation, which validate our model.

Replacing the CCD camera by a calibrated photo-detector, propagation losses of $0.3 \pm 0.1 \mathrm{~dB}^{-\mathrm{cm}^{-1}}$ have been measured with and without an undoped CA cladding, which shows the good optical quality of the CA layer. One of those waveguides was used for all subsequent measurement and will be referred to as wg1. We also characterized a $0.7 \mathrm{~dB} \cdot \mathrm{cm}^{-1}$ waveguide, which will be referred as $\mathrm{wg} 2$.

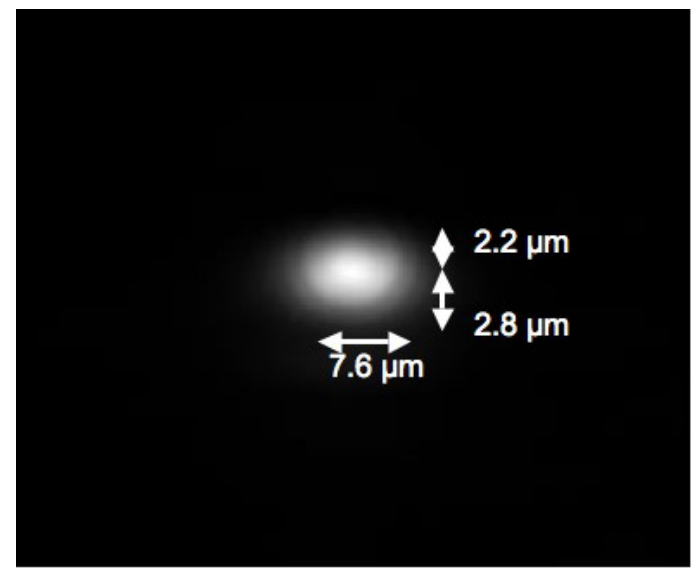

(a)

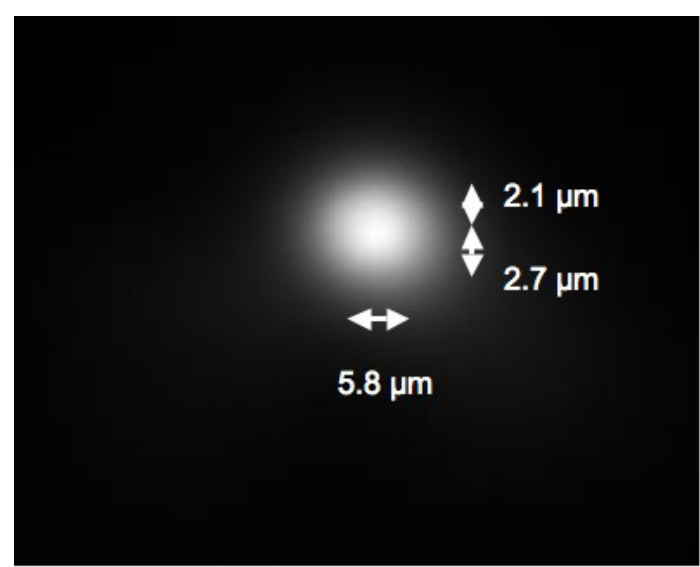

(b)

Figure 5: Measured intensity mode profiles at $\lambda_{\mathrm{s}}=1054 \mathrm{~nm}$ (a) and at $\lambda_{\mathrm{p}}=800 \mathrm{~nm}$ (b)

\section{MODE-LOCKED LASER FINALIZATION AND CHARACTERIZATION}

\subsubsection{PROCESS FLOW}

After realizing the amplifying waveguide, the saturable absorber is deposited on the substrate as it is depicted on figure 6a. First, a mixture of BDN and CA in an acetone solution is prepared in order to have a concentration of $1 \times 10^{18}$ molecules of BDN per $\mathrm{m}^{3}$ into the CA layer after polymerization. Secondly, the layer is deposited to be $100 \mu \mathrm{m}$ thick, which is considered as infinite since only the evanescent wave is in interaction with it. Finally, the layer is dried in a furnace at $150^{\circ} \mathrm{C}$ under vacuum until complete polymerization. Finally, a $99.99 \%$ reflectivity dielectric mirror is stuck on the input facet and another one with a $80 \%$ reflectivity is stuck on the output facet as shown on figure $6 \mathrm{~b}$.

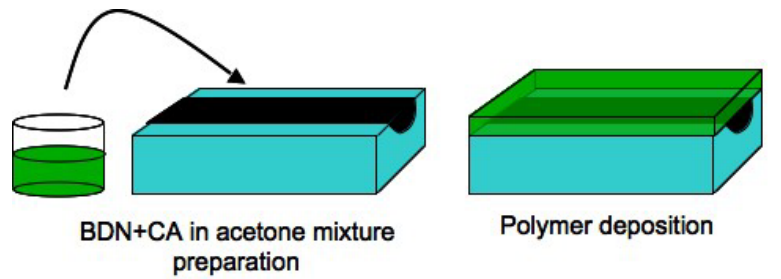

(a)

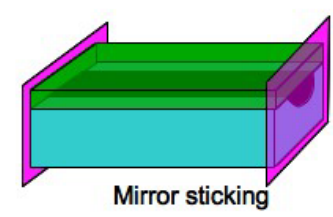

(b)

Figure 6: Saturable absorber preparation and deposition (a) and mirror sticking (b)

\subsection{REPETITION RATE CHARACTERIZATION}

Then, the device, which has been realized, has been characterized with a fast photodiode and an oscilloscope in order to verify the continuous mode-locking operation as depicted of figure 7. 


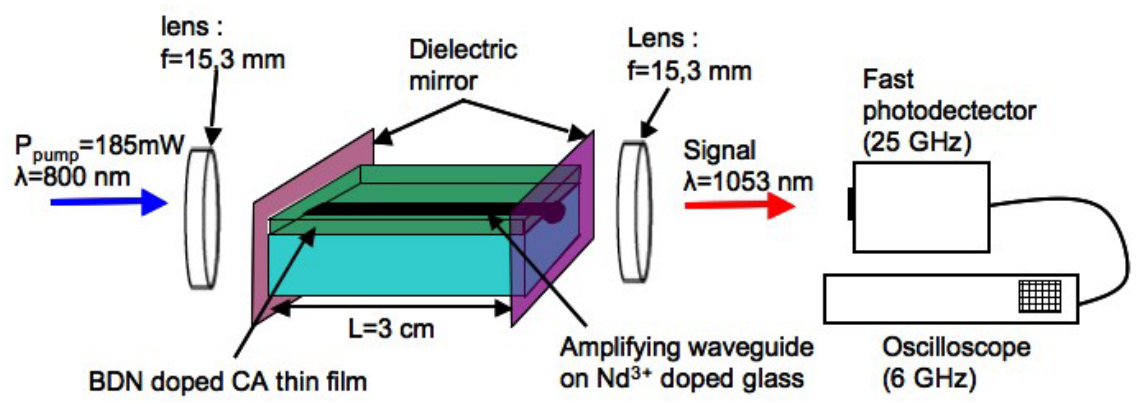

Figure 7: Schematic view of the characterization bench for repetition rate measurement.

For this characterization, the laser was pumped with a Sapphire-Titan laser that has been injected into the amplifying waveguide. The injected power has been previously measured at $185 \pm 5 \mathrm{~mW}$ at $\lambda_{\mathrm{p}}=800 \mathrm{~nm}$. Then the output signal at $\lambda_{\mathrm{s}}=1053 \mathrm{~nm}$ has been focalized on a $25 \mathrm{GHz}$ photodiode linked to a $6 \mathrm{GHz}$ oscilloscope. As we can see on figure 8 , this method is useful for repetition rate characterization, which is in our case equal to 300 ps. This repetition rate corresponds to the photon round-trip time in the $3 \mathrm{~cm}$ laser cavity in the glass medium.

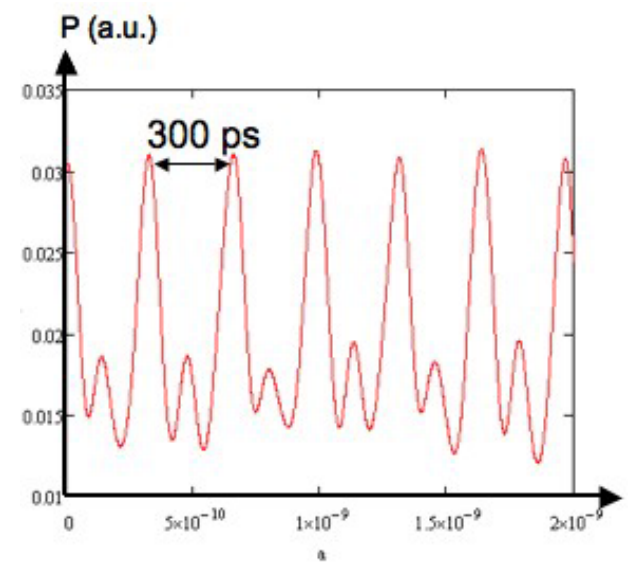

Figure 8: Oscilloscope trace of the output pulse train.

Now, there is still to determine the output pulse width because this method was not quick enough. Actually, the only way to determine the pulse width is the autocorrelation method.

\subsection{PULSE WIDTH AND SPECTRUM CHARACTERIZATION}

First, the wg2 waveguide, which was presented $0.7 \mathrm{~dB} / \mathrm{cm}$ losses, has been characterized. It was emitting $10 \pm$ $0.5 \mathrm{~mW}$ average power when pumped with $185 \mathrm{~mW}$ injected pump power. For characterizing the pulse shape, the fast photodiode of the bench on figure 8 has been replaced by an autocorrelator, which consists of Michelson interferometer and a CCD camera to visualize the visibility of the fringes that depends of the superposition of the pulses at the output of the Michelson interferometer. As depicted on figure 9a, the pulse temporal FWHM width of the autocorrelation trace has been measured to $2 \pm 0.2 \mathrm{ps}$, which corresponds to a computed width of $1.3 \pm 0.2$ ps assuming a sech ${ }^{2}$-shape ${ }^{[11]}$. We can underline than the corresponding spectrum of these pulses, which is depicted on figure $9 \mathrm{~b}$, consists of one $3 \mathrm{~nm}$ wide gate-shaped peak located around a wavelength of $1053 \mathrm{~nm}$. This spectrum has a typical shape of single wavelength emission of a dissipative soliton operation mode-locked laser as described by Zhang et al. ${ }^{[15]}$. 


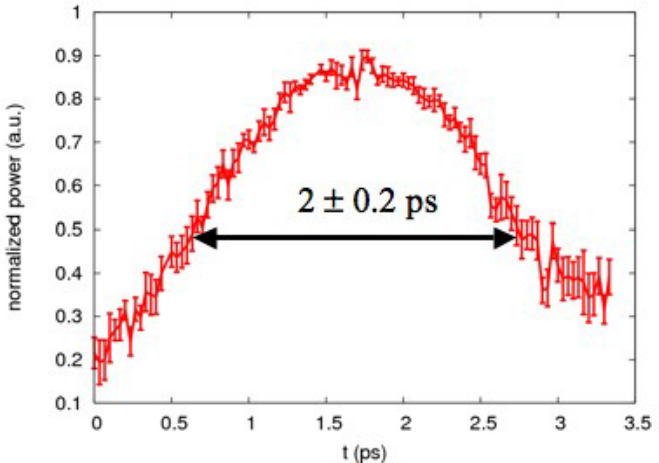

(a)

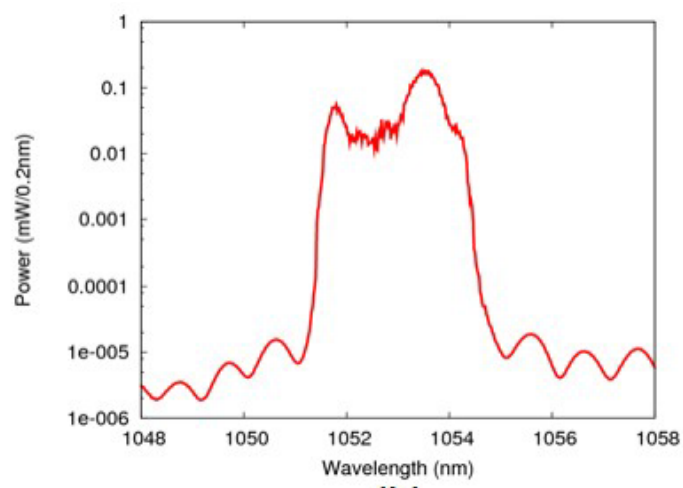

(b)

Figure 9: Autocorrelation trace (a) and spectrum (b) of a single wavelength pulse (laser wg2).

Then, for the laser based on waveguide wg1, double-frequency dissipative soliton pulses have been observed. Indeed, the spectrum presented on figure $10 \mathrm{~b}$ clearly contains two gate-shaped peaks located around a wavelength of $1053 \mathrm{~nm}$. From the autocorrelation trace presented on figure 10a, a temporal FWHM width has been measured to $3.5 \pm 0.2 \mathrm{ps}$, which corresponds to a computed width of $2.3 \pm 0.2 \mathrm{ps}$. For this device, the average output power $30 \pm 0.5 \mathrm{~mW}$ when pump with a $185 \mathrm{~mW}$ injected pump power. The interference pattern in the autocorrelation function confirms the coherent dual-wavelength nature of the pulse. It can be noticed that the $0.4 \mathrm{ps}$ period of the autocorrelation pattern (fig 10a) corresponds to the $2.2 \mathrm{~nm}$ or $600 \mathrm{GHz}$ spacing between the two peaks of the spectrum (fig. 10b).

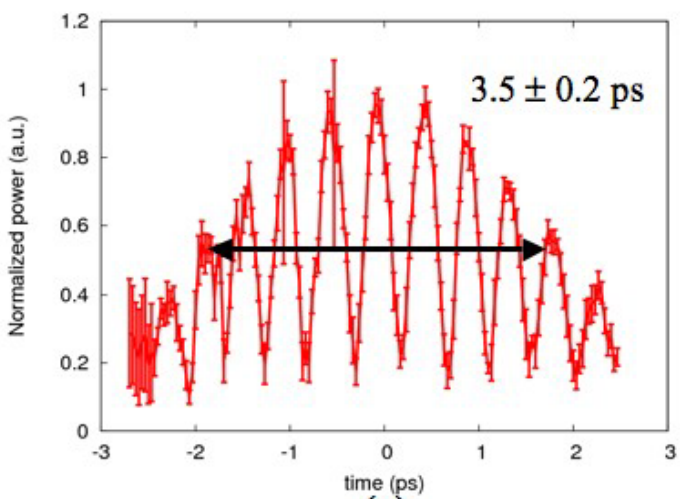

(a)

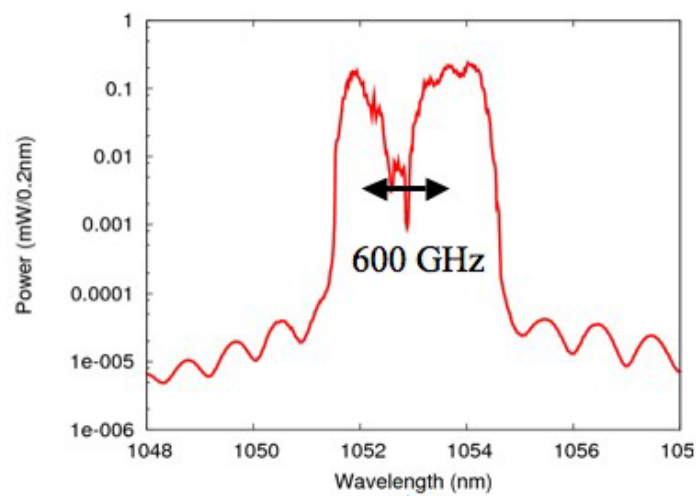

(b)

Figure 10: Autocorrelation (a) and spectrum (b) of a double wavelength pulse (laser wg1).

\section{DISCUSSION}

We can underline the fact that BDN concentration is very low in this device. Indeed, saturable losses are computed to be $0.01 \mathrm{~dB} / \mathrm{cm}$, which is very weak compare to the small signal gain of $\mathrm{dB} / \mathrm{cm}$. We consequently can assume than the pulse formation is not only due to the saturable absorber, but other phenomena have to be taken into account for the modeling the pulse shaping in this kind of mode-locked laser. Indeed, if we model passive mode-locking following this equation (3), the saturable absorber losses parameter is very weak compare to the gain and constant losses ones so that it can be neglected in this model.

$$
\frac{\partial A(z, t)}{\partial z}=(g(z, t)-q(z, t)-l) \cdot A(z, t)
$$


Where $A(z, t)$ is the complex pulse envelop, which depend on the position in the laser cavity $\mathrm{z}$ following the pulse envelop at time $\mathrm{t}, g(z, t)$ is the gain in position $\mathrm{z}$ at time $\mathrm{t}, q(z, t)$ is the saturable absorption in position $\mathrm{z}$ at time $\mathrm{t}$ and $l$ is the constant loss parameter, which represents the propagation losses and the mirror losses.

Indeed, because of the wave confinement characteristic of the laser in a glass medium, two other phenomena have to be taken into account. First, the GVD parameter, which depend of the confinement of the guided mode as well as the waveguide media, is described in equation (4):

$$
\frac{\partial A(z, t)}{\partial z}=-i \cdot D_{2} \cdot \frac{\partial^{2} A(z, t)}{\partial t^{2}}
$$

Where $\mathrm{D}_{2}$ is the chromatic dispersion parameter of the waveguide.

In our case, the mode profile being similar than in optical fiber and the medium being phosphate glass, we can consider than we are in all normal dispersion operation with the parameter $\mathrm{D}_{2}>0$ in equation (4) like in phosphate optical fiber. Moreover, in mode-lock operation regime, the power density is as high as the non-linear refractive index of the phosphate glass is no more negligible. Indeed, the refractive index of the glass $n$ is:

$$
n=n_{0}+I \cdot n_{2}
$$

Where $n_{0}$ is the constant refractive index of the medium ( $n_{0}=1.52$ in our case), $I$ is the intensity of the guided mode and $n_{2}$ is the non-linear refractive index of the medium $\left(n_{2}=3.2 \times 10^{-20} \mathrm{~m}^{2} / \mathrm{W}\right.$ in phosphate glass $\left.{ }^{[19]}\right)$.

This coefficient stimulate the effects of SPM in our waveguide in this form:

$$
\frac{\partial A(z, t)}{\partial z}=i \cdot \gamma \cdot|A(z, t)|^{2} \cdot A(z, t)
$$

Where $\gamma=\frac{2 \pi \cdot n_{2} \cdot c}{n_{0} \cdot \lambda_{s} \cdot A_{\text {eff }}}$ is the non-linear coefficient, which depends of the non-linear refractive index $n_{2}$, of the light velocity under vacuum $c$, of the signal wavelength $\lambda_{s}$ and of the effective area of the guided mode $A_{\text {eff }}$.

Considering all these parameters and knowing than we are in an all-normal GVD mode of operation, the two phase phenomena, which the SPM and the GVD, are of the same sign so that they are not compensating each other. Consequently, another parameter has to be taken into account. Indeed, we have to consider the spectral filtering of the gain bandwidth, which will stabilize the mode-locking operation. This spectral filter can be consider like a parabolic attenuation as it is implemented in the non-linear Schrödinger equation (7):

$$
\frac{\partial A(z, t)}{\partial z}=(g(z, t)-q(z, t)-l) \cdot A(z, t)+i \cdot\left(\gamma \cdot|A(z, t)|^{2}+D_{g} \cdot \frac{\partial^{2}}{\partial t^{2}}-D_{2} \cdot \frac{\partial^{2}}{\partial t^{2}}\right) \cdot A(z, t)
$$

Where $D_{g}$ is the half-width of the medium gain bandwidth.

This working principle is so called dissipative soliton mode-locking operation. This kind of operation mode produced highly chirped ${ }^{[13]}$ pulses and it also can produce multi-wavelength pulses ${ }^{[15]}$. Moreover, the dissipative soliton non-linear Schrödinger equation determines a hyperbolic secant pulse shape ${ }^{[11]}$, which could be useful to know pulse width determination after autocorrelation measurement.

\section{PERSPECTIVES AND CONCLUSION}

In this paper, we presented the complete design, theoretical behavior and characteristics of a dissipative soliton operation in a fully integrated mode-locked laser. First, ion-exchange based technology made us able to elaborate a design in order obtain low loss and single mode amplifying waveguides. Then a theoretical study of the working principle of dissipative soliton allow us to understand the phenomena that have to be taken into account for the design of our device, which could still be optimized for having more non-linear effects or a 
negative dispersion for compensative soliton generation. Moreover, numerical simulation using the non-linear Schrödinger equation for pulse shape prediction is under development in order to optimize the waveguide design. Finally, primary results that shows the dissipative soliton operation in the previously fabricated device, have been obtained. First, using a cavity with average losses, single-wavelength pulses of 1.3 ps width have been observed. The dissipative soliton operation predicts highly chirped pulses. An experiment to compress them by dechirping with a negative dispersion waveguide or optical fiber is under progress. Secondly, using low-losses waveguides, double frequency pulses have been characterized. This kind of pulse could be interesting for $\mathrm{THz}$ generation. Indeed, the two frequencies of the pulses are coherent, have a high peak power and are spaced by $600 \mathrm{GHz}$, which is close to the terahertz frequency domain. Finally, a triple wavelength pulse has been observed for a very low loss waveguide, so we think that under higher pump power, high order multi-wavelength operation is possible. Such pulses may be interesting for WDM optical communication systems.

\section{REFERENCES}

[1] J. E. Broquin, "Glass integrated optics: state of the art and position toward other technologies," in Proceedings of SPIE 6475, p. 647507 (2007).

[2] B. Buchold, C. Glingener, D. Culemann, et E. Voges, "Polarization Insensitive Ion-Exchanged ArrayedWaveguide Grating Multiplexers in Glass," Fiber And Integrated Optics 17, 279-298 (1998).

[3] D. Bucci, J. Grelin, E. Ghibaudo, et J. E. Broquin, "Realization of a 980-nm/1550-nm pump-signal (de) multiplexer made by ion-exchange on glass using a segmented asymmetric y-junction," IEEE Photonics Technology Letters 19, 698-700 (2007).

[4] S. El-Sabban, D. Khalil, I. Schanen, et P. Benech, "Design of an integrated optical magic T for astronomy applications," Applied Optics 39, 6781-6786 (2000).

[5] S. Olivier, L. Delage, F. Reynaud, V. Collomb, M. Trouillon, J. Grelin, I. Schanen, V. Minier, J. E. Broquin, et al., "MAFL experiment: development of photonic devices for a space-based multiaperture fiber-linked interferometer," Applied optics 46, 834-844 (2007).

[6] G. C. Righini, S. Pelli, M. Brenci, M. Ferrari, C. Duverger, M. Montagna, et R. Dall'Igna, "Active optical waveguides based on Er-and Er/Yb-doped silicate glasses," Journal of Non-Crystalline Solids 284, 223229 (2001).

[7] F. Gardillou, L. Bastard, et J. E. Broquin, " $4.25 \mathrm{~dB}$ gain in a hybrid silicate/phosphate glasses optical amplifier made by wafer bonding and ion-exchange techniques," Applied Physics Letters 85, 5176 (2004).

[8] D. Barbier, J. M. Delavaux, T. A. Strasser, M. Rattay, R. L. Hyde, P. Gastaldo, et A. Kevorkian, "Subcentimeter length ion-exchanged waveguide lasers in Er/Yb doped phosphate glass" (1997).

[9] L. Bastard, J. E. Broquin, F. Gardillou, C. Cassagnettes, J. P. Schlotterbeck, et P. Rondeau, "Development of a ion-exchanged glass integrated optics DFB laser for a LIDAR application," dans Proceedings of SPIE 7218, p. 721817 (2009).

[10] R. Salas-Montiel, L. Bastard, G. Grosa, et J. E. Broquin, "Hybrid Neodymium-doped passively Qswitched waveguide laser," Materials Science \& Engineering B 149, 181-184 (2008).

[11] H. A. Haus, C. V. Shank, et E. P. Ippen, "Shape of passively mode-locked laser pulses," Optics Communications 15, 29-31 (1975).

[12] D. Pudo, H. Byun, J. Chen, J. Sickler, F. X. Klärtner, et E. P. Ippen, "Scaling of passively mode-locked soliton erbium waveguide lasers based on slow saturable absorbers," Opt. Express 16, 19221-19231 (2008).

[13] W. H. Renninger, A. Chong, et F. W. Wise, "Dissipative solitons in normal-dispersion fiber lasers," Physical Review A 77, 23814 (2008).

[14] A. Cabasse, B. Ortaç, G. Martel, A. Hideur, et J. Limpert, "Dissipative solitons in a passively modelocked Er-doped fiber with strong normal dispersion," Optics Express 16, 19322-19329 (2008).

[15] H. Zhang, D. Y. Tang, X. Wu, et L. M. Zhao, "Multi-wavelength dissipative soliton operation of an erbium-doped fiber laser," Optics Express 17, 12692-12697 (2009).

[16] A. Lupascu, A. Kevorkian, T. Boudet, F. Saint-André, D. Persegol, et M. Levy, "Modeling ion exchange in glass with concentration-dependent diffusion coefficients and mobilities," Optical Engineering 35, 1603 (1996).

[17] W. H. Press, S. A. Teukolsky, W. T. Vetterling, et B. P. Flannery, Numerical Recipes in C: The Art of 
Scientific Computing (; Cambridge, Cambridge Univ. Press (1992).

[18] M. L. Huggins, K. H. Sun, et D. O. Davis, "The dispersion of silicate glasses as a function of composition. II," JOSA 32, 635-648 (1942).

[19] P. Polynkin, A. Polynkin, D. Panasenko, N. Peyghambarian, et J. Moloney, "All-fiber picosecond laser system at $1.5 \mathrm{~m}$ based on amplification in short and heavily doped phosphate-glass fiber," IEEE Photon. Technol. Lett 18, 2194-6 (2006). 